\title{
Impact of donor factors on post-transplant delayed recovery of graft function in deceased donor kidney transplantation
}

\author{
Woo Yeong Park ${ }^{1}$, Ohyun Kwon ${ }^{1}$, Yaerim Kim ${ }^{1}$, Jin Hyuk Paek ${ }^{1}$, Kyubok Jin ${ }^{1}$, Young-Nam Roh ${ }^{2}$, Ui Jun Park ${ }^{2}$, \\ Hyoung Tae Kim², Seungyeup $\mathrm{Han}^{1}$ \\ 1Division of Nephrology, Department of Internal Medicine, Keimyung University Dongsan Medical Center, Daegu, Korea
${ }^{2}$ Division of Transplantation, Department of Surgery, Keimyung University Dongsan Medical Center, Daegu, Korea
}

Background: Deceased donor (DD) factors influence absolutely the post-transplant delayed recovery of graft function (DGF) in deceased donor kidney transplantation (DDKT). Although there are some tools for the status of DD such as expanded criteria donor criteria or kidney donor profile index score, but these tools do not fully reflect impact of DD factors on post-transplant DGF. Therefore, we aimed at investigating the DD factors related with the occurrence of DGF in DDKT.

Methods: We retrospectively analyzed the medical records of DDKT performed at Keimyung University Dongsan Medical Center between February 2014 and September 2019. Our study enrolled 186 kidney transplant recipients (KTRs), with median follow-up of 45 months after DDKT.

Results: Eighty-seven DDs (46.5\%) had a documented history of smoking; 108 (57.8\%), proteinuria; 80 (42.8\%), transfusion; 95 (50.8\%), acute kidney injury (AKI); and continuous renal replacement therapy (CRRT), 18 (9.6\%). The incidence of DGF was significantly higher in KTRs from DDs with CRRT compared to those from DDs without CRRT $(P=0.002)$. The incidence of DGF was also higher in KTRs from DDs with smoking history, but there were no significant association with the presence and amount of proteinuria and transfusion. Allograft function was significantly lower in the KTRs with DGF until 1 month after KT compared to those without DGF, but there was no significant difference of allograft function between the two groups at 12 months after KT. In Kaplan-Meier analysis, graft and patient survivals did not show the differences according to the characteristics of DDs with smoking, proteinuria, transfusion, AKI, and CRRT. In multivariate logistic analysis, AKI, CRRT, smoking, and donor hypertension were independent risk factors for DGF.

Conclusions: DDs with AKI, CRRT, smoking, and hypertension can increase the risk of occurrence of DGF in KTRs. Therefore, DDKT from DDs with significant risk factors should be performed carefully.

Corresponding author: Woo Yeong Park

E-mail:wy-my@hanmail.net

(c) The Korean Society for Transplantation

This is an Open Access article distributed under the terms of the Creative Commons Attribution Non-Commercial License (http://creativecommons.org/licenses/by-nc/4.0/) which permits unrestricted non-commercial use, distribution, and reproduction in any medium, provided the original work is properly cited. 